Background/introduction National HIV Testing Week (NHTW) aims to increase the earlier detection and treatment of HIV by increasing access to testing across community and statutory settings, with a focus on at risk populations including men who have sex with men (MSM). However there are increasing concerns about risky behaviour, including Chemsex, and an increase in other STIs in MSM.

Aims/objectives To review the acceptance by MSM, of full sexually transmitted infection (STI) screening in a community setting, during NHTW.

Methods We promoted NHTW using national and local material, shared across social media platforms aimed at MSM. In addition to HIV point of care testing (POCT) using a 4th generation test, we offered full STI screening (urine and self-taken pharyngeal/rectal swabs for chlamydia and gonorrhoea NAATs and syphilis POCT). Sexual histories were self-completed.

Results 74 patients were screened; 56 identified as MSM; average age $33(17-75)$. Of these only $21(38 \%)$ reported consistent condom use for anal sex. $4(7 \%)$ reported Chemsex, with MCAT the commonest drug. 20(36\%) had a past history of an STI. 42(75\%) underwent full screening, 12(21\%) POCT only (10 HIV and syphilis, $2 \mathrm{HIV}$ ), and 2(4\%) for chlamydia and gonorrhoea only. There were 3 positive diagnoses: $1 \mathrm{HIV}, 1$ pharyngeal gonorrhoea and 1 rectal chlamydia.

Discussion/conclusion NHTW has proven its effectiveness in increasing the uptake of HIV testing in at risk populations; we have shown that offering full sexual health screening as part of NHTW activity, using self-taken history and urine / non-invasive swabs, is acceptable and effective.

\section{P211 MSM THE COST OF HAVING A GOOD TIME? A SURVEY ABOUT SEX, DRUGS AND LOSING CONTROL}

Nicky Dearing ${ }^{*}$, Sarah Flew. Nottingham University Hospitals NHS Trust, Nottingham, UK

\subsection{6/sextrans-2015-052126.255}

Background Men who have sex with men (MSM) suffer substantial health inequalities compared to the rest of the population relating to sexual health, mental health and drug use. Increasing substance misuse in a sexualised context (chemsex) has been linked to risky sexual behaviour and STI acquisition.

Aims Determine the prevalence of chemsex in our local MSM population, and associated risks to sexual health.

Methods Men attending a GU clinic during December 2014, who identified as MSM, were invited to complete an anonymous questionnaire. 53 questionnaires were received.

Results Overall, 53\% reported some form of recreational drug use. $38 \%$ reported having chemsex. Chemsex participants were more likely to use mephodrone and Viagra than ecstasy and cocaine used more frequently by other party drug users. $47 \%$ of MSM surveyed used the internet to meet partners. The number of partners (any kind of sexual contact) was similar for MSM using drugs and those not. Unprotected receptive anal sex, including with a partner of unknown HIV status, was higher for MSM reporting chemsex. Men reporting chemsex were less likely to have an up-to-date HIV test $(40 \%$ untested in previous year). Overall $40 \%$ reported having an STI in the last year (most commonly Gonorrhoea). All those receiving an HIV diagnosis in the last year $(n=3)$ were amongst the chemsex group. $49 \%$ reported a mental health problem, with $60 \%$ of chemsex participants having a history of depression and/or anxiety.
Conclusion Tackling the sexual health inequalities of MSM is complex, with substance misuse, social media, and mental wellbeing having an increasing influence.

\section{\begin{tabular}{|l|l}
\hline P212 DO YOU KNOW WHO THE MALE SEX WORKERS ARE IN \\
\hline
\end{tabular} YOUR COHORT?}

${ }^{1}$ Sarah Stockwell*, ${ }^{1}$ Gillian Dean, ${ }^{2}$ Marc Tweed, ${ }^{2}$ Tom Boyt. ${ }^{1}$ Brighton and Sussex University Hospitals NHS Trust, Brighton, UK; ${ }^{2}$ Terrance Higgins Trust, Brighton, UK

\section{$10.1136 /$ sextrans-2015-052126.256}

Background/introduction Pro(TECT) is a bespoke service for male sex workers (MSW) launched by THT in June 2014. The service provides MSW with point of care HIV tests, STI and blood borne virus screening, as well as motivational interviewing, harm reduction and signposting to local SH services.

Aim(s)/objectives To describe the sexual health of MSW engaging with the local GU clinic.

Methods The notes of the Pro(TECT) clients who attended the local GU clinic were reviewed.

Results 15 MSW aged 20-57 years, attended the Pro(TECT) service from June to December 2014. 87\% (13/15) had ever attended the GU clinic; 10 in the last 12-months. Only 3 revealed they were MSW. 39\% (5/13) were HIV positive, of whom 3 had detectable viral loads (42,000 to 307,000 copies/ $\mathrm{ml})$. CD4 counts ranged from $6-698 \times 10^{6} / 1.85 \%$ were hepatitis B immune; 1 was hepatitis C co-infected (viral load 100, $4492 \mathrm{cp} / \mathrm{ml})$.

33 STI screens were performed in the last 12 -months, with an average of 3 screens/person. 39\% (5/13) had an acute STI: 4 rectal, 3 pharyngeal, 1 urethral gonorrhoea; 2 rectal chlamydia; 1 latent (early/late) syphilis. PEP was used by 2 of the 5 HIV negative MSW a total of 5 times in 12-months.

Discussion/conclusion There is a high burden of STIs in this group and a significant risk of onward transmission. MSW may not disclose their work to health care professionals (HCP), even on direct questioning. Identification of MSW is fundamental in order to reduce risk and minimise harm. Pro(TECT) acts as a unique gateway into mainstream services, including advice to access PEP.

\section{P213 PRO(TECT) SERVICE - ENGAGING WITH MALE SEX WORKERS}

${ }^{1}$ Sarah Stockwell*, 'Gillian Dean, ${ }^{2}$ Mark Tweed, ${ }^{2}$ Tom Boyt. 'Brighton and Sussex University Hospitals NHS Trust, Brighton, UK; ${ }^{2}$ Terrance Higgins Trust, Brighton, UK

\subsection{6/sextrans-2015-052126.257}

Background/introduction Pro(TECT), a bespoke service for male sex workers (MSW), was launched in June 2014 by THT to engage with local high-risk MSM. Motivational interviewing (MI) is used to explore behaviour change; clients are offered a wide range of support to improve sexual health and reduce onward STI/HIV transmission. Analysis of behaviour and service evaluation was performed at 6 months.

Methods All clients (15) completed an online survey regarding sexual practices, drug/alcohol use and experience of the service.

Results In the last 12 months: 70\% reported insertive UAI; 40\% receptive UAI; 36\% diagnosed with an STI; 29\% HIV positive; $21 \%$ injected drugs; $43 \%$ under the influence of drugs/alcohol while selling sex; 33\% self-harmed in last 12 months. 
Abstract P213 Table 1 Feedback on Ml sessions (33 interventions)

\begin{tabular}{lll}
\hline & $\begin{array}{l}\text { Strongly } \\
\text { Agree/Agree }\end{array}$ & Not Agree \\
\hline "My knowledge on SH has improved" & $80 \%$ & $20 \%$ \\
"My confidence to look after my SH has improved" & $80 \%$ & $20 \%$ \\
"My motivation to look after my SH has improved" & $93 \%$ & $7 \%$ \\
"My knowledge on the different ways I can test for & $69 \%$ & $31 \%$ \\
HIV/STIs has improved" & & \\
"My knowledge of PEP/where to access has improved" & $54 \%$ & $46 \%$ \\
"My confidence in managing or abstaining from & $93 \%$ & $7 \%$ \\
drugs/alcohol; making better choices with regard to & & \\
my SH has improved" & & \\
\hline
\end{tabular}

Conclusions Unprotected sex is common among MSW. Early MI results show good improvement in knowledge and risk taking behaviour. High levels of drug/alcohol use and self-harm require close links to mental health services. Pro(TECT) is unique in accessing this 'hard-to-reach' population and offers a holistic service of harm reduction.

\section{P214 ARE WE MISSING SOMETHING? EXTRA-GENITAL CT/GC NAAT TESTING IN FEMALE PATIENTS ATTENDING A YOUNG PERSONS CLINIC}

Elizabeth Williams, Sarah Ramsay*, Sarah Creighton, Tristan Barber. Homerton University Hospital, London, UK

\subsection{6/sextrans-2015-052126.258}

Background/introduction Our service has a dedicated Young Persons Clinic (YPC) for women age $\leq 25$. Current policy is to only offer routine vulvo-vaginal (VVS) or cervical CT/GC NAAT swabs for female patients but we are aware that STIs in non-genital sites may therefore be missed. From 15/04/14 we offered female patients attending our YPC VVS/cervical and extragenital (throat and rectum) swabs, regardless of exposure stated.

Aim(s)/objectives To quantify the number and result of CT/GC extragenital samples from YPC female patients.

Methods NAAT results for all women attending YPC between $15 / 04 / 14-16 / 09 / 14$ were extracted retrospectively from an electronic database held within the clinic.

Results

\begin{tabular}{lll}
\hline & $\begin{array}{l}\text { Number of } \\
\text { patients }\end{array}$ & $\%$ \\
\hline STI Screens & 193 & \\
Acute STI diagnosed at that visit (including TV and PID) & $29 / 193$ & 15 \\
Positive CT/GC NAAT at that visit & $24 / 193$ & 12 \\
More than one site sampled & $34 / 193$ & 18 \\
with positive extragenital CT/GC NAAT *and negative & $4 / 34$ & 12 \\
VVS/cervical CT/GC NAAT & & \\
\hline *GC throat $\times 2$, CT throat $\times 1$, CT throat + GC rectal $\times 1$ & &
\end{tabular}

42 patients were documented to have been offered extragenital swabs. Of those, 34 (81\%) accepted.

Discussion/conclusion Uptake of extragenital site testing was low. This is likely to reflect low rates of offering extragenital swabs, as there was a high rate $(81 \%)$ of acceptance where an offer was documented. Five infections were solely identified from extragenital testing. It is recognised that a positive result does not necessarily imply infection and extragenital tests are currently unlicensed. Therefore this data suggests that further review would be useful.

\section{P215 DELIVERING STI SERVICES IN HOSTELS FOR HOMELESS INDIVIDUALS}

Elizabeth Williams, Sarah Macauley, Sarah Ramsay*, Sarah Creighton. Homerton University Hospital, London, UK

\subsection{6/sextrans-2015-052126.259}

Background/introduction Residents of hostels for homeless individuals have a disproportionate burden of mental and physical health needs, which can expose them to risk of blood born viruses (BBVs) and STIs. Our borough runs 5 hostels which address health and social needs as well as provide accommodation.

Aim(s)/objectives To report on a pilot aiming to improve diagnosis and treatment of BBVs and STIs of residents of these 5 hostels.

Methods Between 14/02/2012 and 14/02/2013 five hostels were visited a minimum of two times. CT/GC NAATs and HIV, Syphilis, Hepatitis B and C serology were offered as well as signposting to other services.

Results

\begin{tabular}{ll}
\hline Number of residents: & \\
- Seen & 56 \\
- With past/current IVDU & $36 / 56(64 \%)$ \\
- With known HIV & $3 / 56(5 \%)$ \\
- With known HCV & $6 / 56(10 \%)$ \\
- Who had previously tested for HIV & $41 / 56(73 \%)$ \\
- Who had tested for HIV in the preceding year & $29 / 56(52 \%)$ \\
- Who had BBV serology on visit & $21 / 56(38 \%)$ \\
- Who had BBV serology on visit that had not previously tested & $8 / 56(14 \%)$ \\
- Who had CT/GC testing on visit & $54 / 56(96 \%)$ \\
New positive diagnoses & $1 / 56(2 \%)-$ HCV \\
\hline
\end{tabular}

Discussion/conclusion Half the residents had been tested for HIV in the preceding year. $14 \%$ had never previously tested for BBV. 38\% accepted BBV testing at this service and 96\% accepted $\mathrm{CT} / \mathrm{GC}$ testing. One new infection was diagnosed. This suggests that existing services meet the needs of the majority of this group. However, this additional service provided support to a minority of individuals who had been unable to negotiate existing services.

\section{P216 MONITORING GENDER RATIO OF GASTROINTESTINAL INFECTION LABORATORY REPORTS AS A MECHANISM FOR IDENTIFYING POSSIBLE INCREASES AMONG MEN WHO HAVE SEX WITH MEN, ENGLAND, 2003-2013}

${ }^{1}$ Piers Mook*, 'Sanch Kanagarajah, 'Daniel Gardiner, ${ }^{1,2}$ Marko Kerac, 'Gwenda Hughes, ${ }^{1,3}$ Nigel Field, ${ }^{1,4}$ Noel McCarthy, ${ }^{1}$ lan Simms, ${ }^{1}$ Chris Lane, ${ }^{1}$ Bob Adak, ${ }^{1}$ Paul Crook. ${ }^{1}$ Public Health England, London, UK; ${ }^{2}$ London School of Hygiene and Tropical Medicine, London, UK; ${ }^{3}$ University College London, London, UK; ${ }^{4}$ University of Warwick, London, UK

\subsection{6/sextrans-2015-052126.260}

Background Since 2011, an increase in Shigella flexneri has been observed in men due to faecal-oral transmission associated with sexual contact between men who have sex with men (MSM). Sexual history is not routinely collected for cases of gastrointestinal infections. 